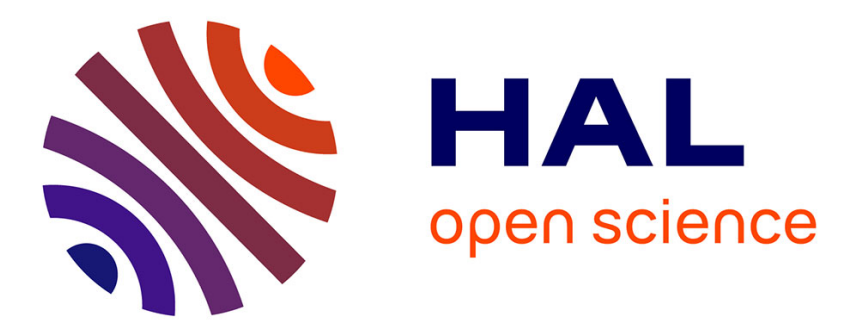

\title{
On Subgraphs of Bounded Degeneracy in Hypergraphs
}

Kunal Dutta, Arijit Ghosh

\section{To cite this version:}

Kunal Dutta, Arijit Ghosh. On Subgraphs of Bounded Degeneracy in Hypergraphs. 42nd International Workshop on Graph-Theoretic Concepts in Computer Science (WG 2016), Jun 2016, Istanbul, Turkey. hal-01360425

\section{HAL Id: hal-01360425 \\ https://hal.science/hal-01360425}

Submitted on 6 Sep 2016

HAL is a multi-disciplinary open access archive for the deposit and dissemination of scientific research documents, whether they are published or not. The documents may come from teaching and research institutions in France or abroad, or from public or private research centers.
L'archive ouverte pluridisciplinaire HAL, est destinée au dépôt et à la diffusion de documents scientifiques de niveau recherche, publiés ou non, émanant des établissements d'enseignement et de recherche français ou étrangers, des laboratoires publics ou privés. 


\title{
On Subgraphs of Bounded Degeneracy in Hypergraphs
}

\author{
Kunal Dutta ${ }^{1}$ and Arijit Ghosh ${ }^{2}$ \\ 1 DataShape, INRIA Sophia Antipolis - Méditerranée, France. \\ duttkunal@gmail. $\operatorname{com}^{\star \star \star}$ \\ 2 ACM Unit, Indian Statistical Institute, Kolkata, India. \\ arijitiitkgpster@gmail. $\mathrm{com}^{\dagger}$
}

\begin{abstract}
A $k$-uniform hypergraph has degeneracy bounded by $d$ if every induced subgraph has a vertex of degree at most $d$. Given a $k$ uniform hypergraph $H=(V(H), E(H))$, we show there exists an induced subgraph of size at least

$$
\sum_{v \in V(H)} \min \left\{1, c_{k}\left(\frac{d+1}{d_{H}(v)+1}\right)^{1 /(k-1)}\right\},
$$

where $c_{k}=2^{-\left(1+\frac{1}{k-1}\right)}\left(1-\frac{1}{k}\right)$ and $d_{H}(v)$ denotes the degree of vertex $v$ in the hypergraph $H$. This extends and generalizes a result of AlonKahn-Seymour (Graphs and Combinatorics, 1987) for graphs, as well as a result of Dutta-Mubayi-Subramanian (SIAM Journal on Discrete Mathematics, 2012) for linear hypergraphs, to general $k$-uniform hypergraphs. We also generalize the results of Srinivasan and Shachnai (SIAM Journal on Discrete Mathematics, 2004) from independent sets (0-degenerate subgraphs) to $d$-degenerate subgraphs. We further give a simple nonprobabilistic proof of the Dutta-Mubayi-Subramanian bound for linear $k$-uniform hypergraphs, which extends the Alon-Kahn-Seymour (Graphs and Combinatorics, 1987) proof technique to hypergraphs. Our proof combines the random permutation technique of Bopanna-Caro-Wei (see e.g. The Probabilistic Method, N. Alon and J. H. Spencer; Dutta-MubayiSubramanian) and also Beame-Luby (SODA, 1990) together with a new local density argument which may be of independent interest. We also provide some applications in discrete geometry, and address some natural algorithmic questions.
\end{abstract}

Keywords: degenerate graphs, independent sets, hypergraphs, and random permutations

\section{Introduction}

For $k \geq 2$, a $k$-uniform hypergraph is a pair $(V(H), E(H))$ where $E(H) \subseteq$ $\left(\begin{array}{c}V(H) \\ k\end{array}\right)$. We will call $V(H)$ and $E(H)$ the vertex set and edge set of $H$ respectively.

*** Kunal Dutta is supported by the European Research Council Advanced Grant 339025 GUDHI (Geometric Understanding in Higher Dimensions).

$\dagger$ Arijit Ghosh is supported by Ramanujan Fellowship, 2016. 
When there is no chance of confusion, we will use $V$ and $E$ to denote $V(H)$ and $E(H)$. For a vertex $v \in V(H)$, degree $d_{H}(v)$ of $V(H)$ will denote $\mid\{e: e \in$ $E(H), v \in e\} \mid$. For readability, $k-1$ will be denoted by $t$.

For a subset $I \subseteq V(H)$, the induced $k$-uniform hypergraph $H(I)$ of $I$ denotes the hypergraph $\left(I, E(H) \cap\left(\begin{array}{l}I \\ k\end{array}\right)\right)$. A hypergraph is linear if every pair of vertices are contained in at most a single hyperedge, i.e. any pair of hyperedges intersect in at most one vertex. A hypergraph $H=(V, E)$ is $d$-degenerate if the induced hypergraph of all subsets of $V$ has a vertex of degree at most $d$, i.e., for all $I \subseteq V$, there exists $v \in I$ such that $d_{H(I)}(v) \leq d$. For a $k$-uniform hypergraph $H=(V, E)$, we will denote by $\alpha_{k, d}(H)$ the size of a maximum-sized subset of $V$ whose induced hypergraph is $d$-degenerate, i.e.,

$$
\alpha_{k, d}(H)=\max \{|I|: I \subseteq V, H(I) \text { is } d \text {-degenerate }\} .
$$

Observe that $\alpha(H):=\alpha_{k, 0}(H)$ is the independence number of the hypergraph $H$.

\subsection{Previous Results}

Turán Tur41 gave a lower bound on the independence number of graphs: $\alpha(G) \geq \frac{n}{d+1}$ where $d$ is the average degree of vertices in $G$.

Caro [Car79] and Wei Wei81] independently showed that for graphs

$$
\alpha(G) \geq \sum_{v \in V(G)} \frac{1}{d_{G}(v)+1},
$$

see AS08. This degree-sequence based bound improves on the original averagedegree based lower bound of Turán, and matches it in the case when all degrees are equal.

For hypergraphs, Spencer Spe72 gave a bound on the independence number, based on the average degree $d: \alpha(H) \geq c_{k}\left(\frac{n}{d^{1 / t}}\right)$, where $c_{k}$ is independent of $n$ and $d$. Caro and Tuza [CT91 generalized the Caro-Wei result to the case of hypergraphs:

Theorem 1. For all $k$-uniform hypergraph $H$, we have

$$
\alpha(H) \geq \sum_{v \in V} \frac{1}{\left(\begin{array}{c}
d_{H}(v)+1 / t \\
d_{H}(v)
\end{array}\right)} .
$$

The above theorem directly implies the following corollary, which gives Spencer's bound:

Corollary 1. For all $k \geq 2$, There exists $d_{k}>0$ such that all $k$-uniform hypergraphs $H$ satisfy

$$
\alpha(H) \geq d_{k} \sum_{v \in V} \frac{1}{\left(1+d_{H}(v)\right)^{1 / t}} .
$$


Further, Thiele [Thi99] obtained a lower bound on the independence number of arbitrary (non-uniform) hypergraphs, in terms of the degree rank, a generalization of the degree sequence.

On the algorithmic side, Srinivasan and Shachnai [SS04, used the random permutation method of Beame and Luby BL90 and also Bopanna-Caro-Wei (see e.g. [AS08, DMS12]), together with the FKG correlation inequality, to obtain a randomized parallel algorithm for independent sets, which matched the asymptotic form of the Caro-Tuza bound. Dutta, Mubayi and Subramanian DMS12 also used the Bopanna-Caro-Wei method; using elementary techniques they obtained degree-sequence based lower bounds on the independence numbers of $K_{r}$-free graphs and linear $k$-uniform hypergraphs, which generalized the earlier average-degree based bounds of Ajtai et al. [AKS80, Shearer [She83, She95] and Duke, Lefmann and Rödl [DLR95], in terms of degree sequences. ${ }^{3}$

Average degree vs. degree-sequence In general, a bound using the degree sequence would be intuitively expected to be better than a bound using just the average degree, since it has more information about the graph. For the above bounds on the independence numbers, this essentially follows from the convexity of the function $x^{-1 / t}$. Dutta-Mubayi-Subramanian [DMS12] gave constructions of hypergraphs which show that the bounds based on the degree-sequence can be stronger than those based on the average degree by a polylogarithmic (in the number of vertices) factor.

Large $\boldsymbol{d}$-degenerate subgraphs Compared to independent sets, $d$-degenerate subgraphs have been less well-investigated. However, it includes as special cases zero-degenerate subgraphs i.e. independent sets, as well as 1-degenerate subgraphs, i.e. maximum induced forests, whose complements are the well-known hitting set and feedback vertex set problems respectively. The best known result on this question is that of Alon, Kahn and Seymour [AKS87, who proved the following lower bound for $\alpha_{2, d}(G):{ }^{4}$

Theorem 2 ([AKS87]). For all graphs $G=(V, E)$ we have

$$
\alpha_{2, d}(G) \geq \sum_{v \in V} \min \left\{1, \frac{d+1}{d_{G}(v)+1}\right\} .
$$

This bound is sharp for every $G$ which is a disjoint union of cliques. Moreover, they gave a polynomial time algorithm that finds in $G$ an induced d-degenerate subgraph of at least this size.

${ }^{3}$ Their proof also yields an elementary proof of the main bound of Srinivasan and Shachnai SS04] without using correlation inequalities, though they do not state this explicitly.

${ }^{4}$ Alon, Kahn and Seymour AKS87 actually defined a $d$-degenerate graph as one where every subgraph has a vertex of degree less than $d$, whereas we use the more usual definition in which every subgraph has a vertex of degree at most $d$. 
On the algorithmic side, Pilipczuk and Pilipczuk [PP12] addressed the question of finding a maximum $d$-degenerate subgraph of a graph, giving the first algorithm with running time $o\left(2^{n}\right)$. Zaker [Zak13] studied a more general version of degeneracy and gave upper and lower bounds for finding the largest subgraph of a 2-uniform graph having a given generalized degeneracy.

The proof of Dutta-Mubayi-Subramanian DMS12 implies the following lower bound on $\alpha_{k, d}$ for linear hypergraphs (though not explicitly stated in their paper):

Theorem 3 ([DMS12]). Let $G=(V, E)$ be a linear $k$-uniform hypergraph, and for all $v \in V, d_{G}(v)$ denote the degree of $v$ in $G$. Then

$$
\alpha_{k, d}(G) \geq w(G):=\sum_{v \in V} w_{G}(v),
$$

where

$$
w(v)= \begin{cases}1 & \text { if } d_{G}(v) \leq d \\
\frac{1}{1+(t(d+1))^{-1}} \frac{\left(\begin{array}{c}
d_{G}(v) \\
d+1
\end{array}\right)}{\left(\begin{array}{c}
d_{G}(v)+1 / t \\
d_{G}(v)-d-1
\end{array}\right)} & \text { if } d_{G}(v)>d .\end{cases}
$$

\section{Our Results}

We first give a completely different and an extremely simple proof of Theorem 3 using a weight function. Our proof follows along the lines of the proof of Theorem 2 due to Alon, Kahn and Seymour AKS87.

Next, we extend Theorem 3 to the case of general hypergraphs:

Theorem 4. Let $G=(V, E)$ be a k-uniform hypergraph, and for all $v \in V$, $d_{G}(v)$ denote the degree of $v$ in $G$. Then

$$
\alpha_{k, d}(G) \geq \sum_{v \in V} \min \left\{1, c_{k}\left(\frac{d+1}{d(v)+1}\right)^{1 / t}\right\},
$$

where $t=k-1$ and $c_{k}=2^{-1-1 /(k-1)}$. There exists a randomized algorithm that can extract a d-degenerate set of above size in expectation.

Our proof uses the random permutation method AS08 of Bopanna-Caro-Wei, together with a new local density argument, avoiding advanced correlation inequalities. As a consequence, we obtain a simpler proof as well as a generalization of the result of Srinivasan-Shachnai [SS04].

As an application of Theorem 4 we will prove the following result in incidence geometry, which generalizes a result of Payne and Wood [PW13] on the maximum size of a subset, out of $n$ points in the plane, such that no three points in the subset are collinear.

Lemma 1. 1. Let $P$ be a set of $n$ points in the plane such that for any line $l$ in the plane $|l \cap P| \leq \ell$. For $d \leq O\left(n \log \ell+\ell^{2}\right)$ there exists a subset $S \subseteq P$ 
with at most $d|S|$ collinear triples in $S$ and

$$
|S|=\Omega\left(\sqrt{\frac{d n^{2}}{n \log \ell+\ell^{2}}}\right) .
$$

And if $\ell \leq O(\sqrt{n})$, then

$$
|S|=\Omega\left(\sqrt{\frac{d n}{\log \ell}}\right) .
$$

2. Let $P$ be a set of $n$ points in the plane such that for any line $l$ in the plane $|l \cap P| \leq \ell$. Let $k \geq 4$ be a constant and $d \leq O\left(\ell^{k-3} n+\ell^{k-1}\right)$. Then there exists a subset $S \subseteq P$ of size

$$
\Omega\left(n\left(\frac{d}{\ell^{k-3} n+\ell^{k-1}}\right)^{1 /(k-1)}\right)
$$

such that $S$ has at most $d|S|$ collinear $k$-tuples in $S$. And if $\ell \leq O(\sqrt{n})$, then

$$
|S|=\Omega\left(\left(\frac{n^{k-2} d}{\ell^{k-3}}\right)^{1 /(k-1)}\right) .
$$

The proof of Lemma1 1 uses the following lemma by Payne and Wood [PW13, proved using Szemerédi-Trotter theorem [ST83] on incidence geometry.

\section{Lemma 2 ([PW13] $)$.}

1. Let $P$ be a set of $n$ points in the plane such that for any line $l$ in the plane $|l \cap P| \leq \ell$. Then the number of collinear 3 -tuples in $P$ is at most $O\left(n^{2} \log \ell+\right.$ $\left.n \ell^{2}\right)$.

2. Let $P$ be a set of $n$ points in the plane such that for any line $l$ in the plane $|l \cap P| \leq \ell$. Then, for $k \geq 4$, the number of collinear $k$-tuples in $P$ is at most $O\left(\ell^{k-3} n^{2}+\ell^{k-1} n\right)$.

Proof (Lemma 1). Let $H$ be a $k$-uniform hypergraph with $V(H)=P$, and $\left\{p_{1}, \ldots, p_{k}\right\} \in E(H)$ if there exists a line a line $l$ in the plane with $\left\{p_{1}, \ldots, p_{k}\right\} \in$ $l$. Lemma 2 bounds the size of $E(H)$. The result now follows directly from Theorem 4

The rest of the paper is organised as follows: Section 2 has the simpler proof of Theorem 3 , and Section 3 has the proof of Theorem 4 Finally in the Conclusions section there are some remarks and open questions. 


\section{Linear Hypergraphs}

In this section we will give an alternative proof of the Theorem 3 . The proof will follow exactly along the lines of the proof by Alon, Kahn and Seymour AKS87. of Theorem 2 ,

First observe that

$$
\frac{\left(\begin{array}{c}
d_{G}(v) \\
r
\end{array}\right)}{\left(\begin{array}{c}
d_{G}(v+1 / t \\
d_{G}(v)-r
\end{array}\right)}=\frac{1}{\left(1+\frac{1}{t(r+1)}\right) \ldots\left(1+\frac{1}{t d_{G}(v)}\right)}
$$

This implies that $w(v)$ is decreasing in $d_{G}(v)$ for all values of $d_{G}(v) \geq r$. Also, observe that

$$
\frac{\left(\begin{array}{c}
d_{G}(v)-1 \\
r
\end{array}\right)}{\left(\begin{array}{c}
d_{G}(v)-1+1 / t \\
d_{G}(v)-1-r
\end{array}\right)}=\left(1+\frac{1}{t d_{G}(v)}\right) \frac{\left(\begin{array}{c}
d_{G}(v) \\
r
\end{array}\right)}{\left(\begin{array}{c}
d_{G}(v)+1 / t \\
d_{G}(v)-r
\end{array}\right)}
$$

The alternative proof will be by induction on the number $n$ of vertices of the $k$-uniform hypergraph $G$. The base case of $n=1$ follows trivially. Assuming the result holds for $n-1$, we will now show that the result also holds for $n$.

\section{Case 1}

If we have a vertex $v \in V(G)$ with $d_{G}(v) \leq d$, then consider the hypergraph $H=G\left(V^{\prime}\right)$ where $V^{\prime}=V \backslash\{v\}$. Observe that $\alpha_{k, d}(G)=\alpha_{k, d}(H)+1$. Since $\forall u \in V^{\prime}$, we have from Equation (4), $w_{G}(u) \leq w_{H}(u)$. This implies

$$
w(H)=\sum_{u \in V^{\prime}} w_{H}(u) \geq \sum_{u \in V^{\prime}} w_{G}(u)=w(G)-1 .
$$

The last inequality follows from the fact that $w_{G}(v)=1$ since $d_{G}(v) \leq d$. Using the induction hypothesis $\alpha_{k, d}(G) \geq w(G)$ and the fact that $\alpha_{k, d}(G)=$ $\alpha_{k, d}(H)+1$, we get

$$
\alpha_{k, d}(G)=\alpha_{k, d}(H)+1 \geq w(H)+1 \geq w(G) .
$$

\section{Case 2}

Now we will consider the case where $d_{G}(v)>d, \forall v \in V(G)$. Let $\Delta=\max _{u \in V(G)} d_{G}(u)$, and let $v \in V(G)$ be a vertex with $d_{G}(v)=\Delta$. Let $u_{1}, \ldots, u_{l}$, where $l=t \Delta$, be the neighbors of $v$ in $G$. Note that $l=t \Delta$ follows from the fact that $G$ is a linear hypergraph. We will now show that $w(H) \geq w(G)$, where $H=G\left(V^{\prime}\right)$ and $V^{\prime}=V \backslash\{v\}$. We will now show $w(H) \geq w(G)$.

$$
\begin{aligned}
w(H) & =\sum_{u \in V^{\prime}} w_{H}(u) \\
& =w(G)-w_{G}(v)-\sum_{i=1}^{l} w_{G}\left(u_{i}\right)+\sum_{i=1}^{l} w_{H}\left(u_{i}\right)
\end{aligned}
$$




$$
\begin{aligned}
& =w(G)-w_{G}(v)+\sum_{i=1}^{l} \frac{w_{G}\left(u_{i}\right)}{t d_{G}\left(u_{i}\right)} \\
& \geq w(G)
\end{aligned}
$$

The second last inequality follows from the facts that $d_{H}\left(u_{i}\right)=d_{G}\left(u_{i}\right)-1$ (as $G$ is a linear hypergraph) and Equation (4). The last inequality follows from the facts that $d_{G}(u) \leq \Delta$ for all $u \in V$ and $w_{G}\left(u_{i}\right) \geq w_{G}(v)$ (direct consequence of Equation (4)). From induction hypothesis we have

$$
\alpha_{k, d}(H) \geq w(H) \geq w(G) .
$$

This completes the proof of Theorem 3 since $\alpha_{k, d}(G) \geq \alpha_{k, d}(H)$.

\section{General $k$-uniform Hypergraphs}

In this section we shall prove a lower bound on $\alpha_{k, d}(H)$ for general $k$-uniform hypergraph $H$ in terms of its degree sequence. We will give a very simple randomized algorithm to obtain an $d$-degenerate subgraph of a $k$-uniform hypergraph, whose analysis in expectation will yield the desired bound in Theorem 4

\subsection{Details of the Algorithm}

Before we can give the details of the algorithm, we will need some definitions.

Definition 1. Let $\sigma$ be an ordering of the vertices of $H$.

- Fix a vertex $v \in V(H)$. Call a hyperedge $e \in E(H)$ with $v \in e$ a backward edge with respect to $\sigma$, if $\forall u \in e \backslash\{v\}, \sigma(u)<\sigma(v)$.

- We will denote by $b_{\sigma}(v)$ the number of backward edges of the vertex $v$ with respect to the ordering $\sigma$.

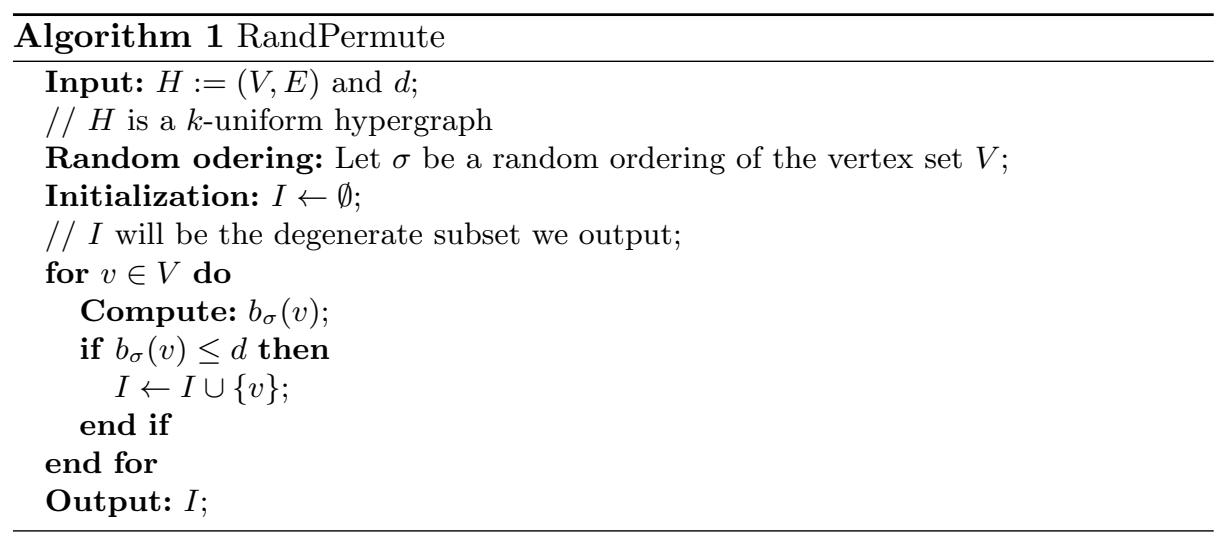




\subsection{Analysis of the Algorithm}

Theorem 4 directly follows from the following result.

Claim

$$
\mathrm{E}[|I|] \geq \sum_{v \in V} \min \left\{1, c_{k}\left(\frac{d+1}{d_{H}(v)+1}\right)^{1 / t}\right\},
$$

where $c_{k}=c_{k}=2^{-\left(1+\frac{1}{k-1}\right)}\left(1-\frac{1}{k}\right)=2^{-\left(1+o_{k}(1)\right)}$.

Proof. For all vertices $v \in V$, we will denote by $N(v)$ the neighbors of $v$ in $H$.

Given an arbitrary vertex $v \in V$, and a random ordering of the vertices $\sigma$, we need to bound $\operatorname{Pr}[v \in I]$ from below. Since the event of $v$ being selected depends on the relative ordering of the vertices in $N(v)$, therefore, the probability $v$ being selected in $I$ in a random ordering is the number of orderings for which $v$ is selected, divided by $(|N(v)|+1)$ !. Let $\sigma$ be an ordering of the vertices of $V$, such that $v$ is selected in $I$ in the ordering $\sigma$. Given a vertex $v \in V$, consider now $L_{v}:=\left(V\left(L_{v}\right), E\left(L_{v}\right)\right)$, the $(k-1)$-uniform link hypergraph on the neighbourhood of $v$, defined as follows:

$$
\begin{gathered}
V\left(L_{v}\right):=N(v), \text { and } \\
E\left(L_{v}\right):=\left\{S \subset V\left(L_{v}\right): S \cup\{v\} \in E\right\},
\end{gathered}
$$

i.e., the vertices are the neighbours of $v$, and the edges are those edges of the original hypergraph $H$ which contained $v$, but with $v$ removed. Clearly $\left|E\left(L_{v}\right)\right|=$ $d_{H}(v)$. Let $F \subset V\left(L_{v}\right)$ be

$$
F:=\{u \in N(v): \sigma(u)<\sigma(v)\},
$$

i.e., the vertices in the neighbourhood of $v$ which occur before $v$ in the ordering $\sigma$. We want $L_{v}(F)$ to have at most $d$ hyperedges. The vertices occurring before $v$ can be ordered arbitrarily amongst themselves, and similarly for the vertices occuring after $v$. So we get that the probability that $v$ is selected in $I$ is given by:

$$
\begin{aligned}
\operatorname{Pr}[v \in I] & =\sum_{J \subset V\left(L_{v}\right):\left|E\left(L_{v}(J)\right)\right| \leq d} \frac{(|J|) !\left(\left|V\left(L_{v}\right)\right|-|J|\right) !}{\left(\left|V\left(L_{v}\right)\right|+1\right) !} \\
& =\frac{1}{\left|V\left(L_{v}\right)\right|+1} \sum_{J \subset V\left(L_{v}\right):\left|E\left(L_{v}(J)\right)\right| \leq d} \frac{1}{\left(\begin{array}{c}
\left|V\left(L_{v}\right)\right| \\
|J|
\end{array}\right)}
\end{aligned}
$$

For $k=2$, the link hypergraph is a 1-graph i.e. a set of vertices, each vertex being a 1-edge. Hence the summation in the RHS evaluates to $d+1$ (counting 1 for each case when there are exactly $0,1, \ldots, d$ vertices before $v$, in the random ordering). Therefore

$$
\mathrm{E}[|I|]=\sum_{v \in V} \operatorname{Pr}[v \in I]=\sum_{v \in V} \min \left\{1, \frac{d+1}{d(v)+1}\right\},
$$


and we get the theorem of Alon-Kahn-Seymour (Theorem 2p).

For general $k$-uniform hypergraphs, observe that if $d_{H}(v) \leq d$, then $\operatorname{Pr}[v \in I]=$ 1. However, if $d_{H}(v)>d$, then we need to look at the link hypergraph which can be an arbitrary $k$-1-uniform hypergraph. In this case, we shall prove the following general lemma, (which may be of independent interest).

Lemma 3. For each $k$-uniform hypergraph $H=(V, E)$, such that $|V|=n$, $|E|=m$, we have

$$
\sum_{J \subset V(H):|E(J)| \leq a} \frac{1}{\left(\begin{array}{c}
n \\
|J|
\end{array}\right)} \geq c_{k}^{\prime} n\left(\frac{a+1}{m}\right)^{1 / k} .
$$

where $c_{k}^{\prime}=2^{-(1+1 / k)}$.

Indeed, we get that the probability that $v$ is selected in $I$ is given by:

$$
\begin{aligned}
\operatorname{Pr}[v \in I] & =\frac{1}{\left|V\left(L_{v}\right)\right|+1} \sum_{J \subset V\left(L_{v}\right):\left|E\left(L_{v}(J)\right)\right| \leq d} \frac{1}{\left(\begin{array}{c}
\left|V\left(L_{v}\right)\right| \\
|J|
\end{array}\right)} \\
& \left.\geq \frac{c_{k-1}^{\prime}\left|V\left(L_{v}\right)\right|}{\left|V\left(L_{v}\right)\right|+1} \times \frac{(d+1)^{1 /(k-1)}}{\left|E\left(L_{v}\right)\right|^{1 /(k-1)}} \quad \text { (from Lemma 3 }\right) \\
& \geq \frac{c_{k-1}^{\prime}\left|V\left(L_{v}\right)\right|}{\left|V\left(L_{v}\right)\right|+1} \times \frac{(d+1)^{1 /(k-1)}}{d_{H}(v)^{1 /(k-1)}} \quad\left(\text { as }\left|E\left(L_{v}\right)\right|=d_{H}(v)\right) \\
& \geq c_{k}\left(\frac{d+1}{d_{H}(v)+1}\right)^{1 /(k-1)},
\end{aligned}
$$

where

$$
c_{k}=2^{-\left(1+\frac{1}{k-1}\right)}\left(1-\frac{1}{k}\right)=2^{-\left(1+o_{k}(1)\right)} .
$$

Note that Inequality (5) follows from the fact that since $d_{H}(v)>d \geq 0$, we must have at least $k-1$ vertices in the hypergraph $L_{v}$, i.e., $\left|V\left(L_{v}\right)\right| \geq k-1$.

It only remains to prove Lemma 3 which we will prove using a local density argument.

Proof (of Lemma 3). For all $1 \leq s \leq n$, we define

$$
\rho_{s}:=\mathrm{E}_{|S|=s}[|E(H(S))|]=\frac{\sum_{S \subseteq V,|S|=s} E(H(S))}{\left(\begin{array}{l}
n \\
s
\end{array}\right)} .
$$

Note that the expectation is taken over all subsets of $V$ of size $s$, and $E(H(S))=$ $\{e \in E(H): e \subseteq S\}$.

Counting the number of pairs $(e, S)$, where $e \in E(H)$, and $S \subset V:|S|=$ $s, e \in S$, in two ways, we get the average local density of sets of size $s$ is

$$
\rho_{s}=\frac{m\left(\begin{array}{l}
n-k \\
s-k
\end{array}\right)}{\left(\begin{array}{l}
n \\
s
\end{array}\right)}=\frac{m(s)_{k}}{(n)_{k}} \leq \frac{m s^{k}}{n^{k}} .
$$


(Here $\left(\begin{array}{l}a \\ b\end{array}\right):=0$ if $\left.b<0\right)$. This is as follows: let

$$
z:=\#\left\{(e, S): e \in E, S \in\left(\begin{array}{c}
V \\
s
\end{array}\right), e \subset S\right\}
$$

Then each of the $\left(\begin{array}{l}n \\ s\end{array}\right)$ sets of size $s$ contributes, on average, $\rho_{s}$-many entries to $z$. On the other hand, each edge $e \in E(H)$ belongs to $\left(\begin{array}{l}n-k \\ s-k\end{array}\right)$-many sets of size $s$. Equating the two summations gives the claimed average local density.

Now, we use the above observation to prove the lemma. Partition the summands on the LHS into $n$ parts, depending on the size of the set $J$ (i.e. the number of neighbouring vertices which precede $v$ in the random ordering):

$$
\sum_{i=1}^{n}\left(\sum_{J \subseteq V:|J|=i,|E(H(J))|<a} \frac{1}{\left(\begin{array}{c}
n \\
i
\end{array}\right)}\right) .
$$

When $i<k$, it is easy to see that the inner summation is 1 . The main idea of the proof is the following: first, observe that for any $i \in[n]$, the inner summation is just the probability that a randomly picked set of exactly $i$ vertices has fewer than $a+1$ edges. Then for small enough $i$, the expected number of edges is upper bounded by $\rho_{i}$, and is much smaller than $(a+1) / 2$. So the probability that a random $i$-set contains more than twice the expected number, is at most half. Therefore for all such $i$, the contribution to the outer sum is at least $1 / 2$. The number of such terms in the outer sum, then gives the claimed lower bound.

Formally, let $X_{i}$ be a random variable giving the number of edges contained in a randomly chosen set on $i$ vertices. By Markov's inequality:

$$
\operatorname{Pr}\left[X_{i} \geq 2 . \mathrm{E}\left[X_{i}\right]\right] \leq \frac{1}{2} .
$$

We have that $\mathrm{E}\left[X_{i}\right]=\rho_{i}$. Therefore, the LHS becomes:

$$
\sum_{i=1}^{n} \operatorname{Pr}\left[X_{i}<a+1\right]=1-\sum_{i=1}^{n} \operatorname{Pr}\left[X_{i} \geq a+1\right]
$$

With foresight, we split the above sum into two parts, when $i \leq t^{*}:=\frac{n(a+1)^{1 / k}}{(2 m)^{1 / k}}$, and when $i>t^{*}$. Observe that when $i \leq t^{*}$, we have that $\frac{m i^{k}}{n^{k}} \leq \frac{a+1}{2}$. We get

$$
\begin{aligned}
\sum_{i=1}^{n} \operatorname{Pr}\left[X_{i}<a+1\right] & =\sum_{i=1}^{t^{*}} \operatorname{Pr}\left[X_{i}<a+1\right]+\sum_{i>t^{*}} \operatorname{Pr}\left[X_{i}<a+1\right] \\
& \geq \sum_{i=1}^{t^{*}}\left(1-\frac{\mathrm{E}\left[X_{i}\right]}{a+1}\right) \\
& =\sum_{i=1}^{t^{*}}\left(1-\frac{m(i)_{k}}{(a+1)(n)_{k}}\right)
\end{aligned}
$$




$$
\begin{aligned}
& \geq \sum_{i=1}^{t^{*}}\left(1-\frac{m i^{k}}{(a+1) n^{k}}\right) \\
& \geq \sum_{i=1}^{t^{*}}\left(1-\frac{1}{2}\right) \\
& \geq \frac{n(a+1)^{1 / k}}{2(2 m)^{1 / k}}
\end{aligned}
$$

where in the second step we used Markov's inequality on the first summation, and in the penultimate step we used the observation on $t^{*}$ noted above.

Remark 1 (Regarding the constant “ $c_{k}$ ").

1. Observe that

$$
c_{k}=2^{-\left(1+\frac{1}{k-1}\right)}\left(1-\frac{1}{k}\right) \geq \frac{1}{8},
$$

for all $k \geq 2$, and $c_{k} \rightarrow \frac{1}{2}$ as $k \rightarrow \infty$.

2. For simplicity of exposition we did not try to optimize $c_{k}$.

\section{Conclusion}

Our randomized algorithm for finding $d$-degenerate subgraphs of $k$-uniform hypergraphs inherits the analysis of Srinivasan-Shachnai [SS04 for independent sets:

(i) The RandPermute algorithm runs in RNC, as long as $d$ is polylogarithmic in the number of vertices and edges.

(ii) Our proof technique generalizes to non-uniform hypergraphs.

(iii) All our results generalize to the vertex-weighted scenario, where we want an induced $d$-degenerate subgraph of maximum weight.

It is interesting to ask if the RandPermute algorithm can be used to obtain lower bounds for the generalized degeneracy of Zaker, or to solve the conjecture of Beame and Luby [BL90], which asks whether iterating the RandPermute algorithm always yields a maximal independent set.

Acknowledgement Kunal Dutta and Arijit Ghosh are supported by European Research Council under Advanced Grant 339025 GUDHI (Algorithmic Foundations of Geometric Understanding in Higher Dimensions) and Ramanujan Fellowship (No. SB/S2/RJN-064/2015) respectively.

Part of this work was done when Kunal Dutta and Arijit Ghosh were Researchers at Max-Planck-Institute for Informatics, Germany, supported by the Indo-German Max Planck Center for Computer Science (IMPECS). 


\section{References}

[AKS80] M. Ajtai, J. Komlós, and E. Szemerédi. A Note on Ramsey Numbers. Journal on Combinatorial Theory, Series A, 29(3):354-360, 1980.

[AKS87] N. Alon, J. Kahn, and P. D. Seymour. Large induced degenerate subgraphs. Graphs and Combinatorics, 3(1):203-211, 1987.

[AS08] N. Alon and J. H. Spencer. The Probabilistic Method. Wiley International, 2008.

[BL90] Paul Beame and Michael Luby. Parallel search for maximal independence given minimal dependence. In Proceedings of the First Annual ACM-SIAM Symposium on Discrete Algorithms, SODA '90, pages 212-218, Philadelphia, PA, USA, 1990. Society for Industrial and Applied Mathematics.

[Car79] Y. Caro. New results on the independence number. Technical report, Tel Aviv University, 1979.

[CT91] Y. Caro and Z. Tuza. Improved lower bounds on $k$-independence. Journal of Graph Theory, 15(1):99-107, 1991.

[DLR95] R. A. Duke, H. Lefmann, and V. Rödl. On Uncrowded Hypergraphs. Random Structures \&6 Algorithms, 6(2/3):209-212, 1995.

[DMS12] K. Dutta, D. Mubayi, and C. R. Subramanian. New Lower Bounds for the Independence Number of Sparse Graphs and Hypergraphs. SIAM Journal on Discrete Mathematics, 26(3):1134-1147, 2012.

[PP12] Marcin Pilipczuk and Michał Pilipczuk. Parameterized and Exact Computation: 7th International Symposium, IPEC 2012, Ljubljana, Slovenia, September 12-14, 2012. Proceedings, chapter Finding a Maximum Induced Degenerate Subgraph Faster Than 2 n, pages 3-12. Springer Berlin Heidelberg, Berlin, Heidelberg, 2012.

[PW13] M. S. Payne and D. R. Wood. On the General Position Subset Selection Problem. SIAM J. Discrete Math., 27(4):1727-1733, 2013.

[She83] J. B. Shearer. A note on the independence number of triangle-free graphs. Discrete Mathematics, 46(1):83-87, 1983.

[She95] J. B. Shearer. On the Independence Number of Sparse Graphs. Random Structures \& Algorithms, 7(3):269-272, 1995.

[Spe72] J. H. Spencer. Turán's theorem for $k$-graphs. Discrete Mathematics, 2:183186, 1972.

[SS04] Hadas Shachnai and Aravind Srinivasan. Finding large independent sets in graphs and hypergraphs. SIAM J. Discrete Math., 18(3):488-500, 2004.

[ST83] E. Szemerédi and W. T. Trotter. Extremal problems in discrete geometry. Combinatorica, 3(3):381-392, 1983.

[Thi99] Torsten Thiele. A lower bound on the independence number of arbitrary hypergraphs. Journal of Graph Theory, 30(3):213-221, 1999.

[Tur41] P. Turán. On an extremal problem in graph theory [in Hungarian]. Math Fiz. Lapok, 48, 1941.

[Wei81] V. K. Wei. A lower bound on the stability number of a simple graph. Technical Memorandum TM 81-11217-9, Bell Laboratories, 1981.

[Zak13] Manouchehr Zaker. Generalized degeneracy, dynamic monopolies and maximum degenerate subgraphs. Discrete Applied Mathematics, 161(1617):2716 - 2723, 2013. 